\title{
Editorial
}

\section{Ulrich Rendtel}

Online publiziert: 27. Januar 2012

(c) Springer 2012

Geburtstage sind immer gut für eine Rückschau und einen Blick in die Zukunft. Aus Anlass des 100. Geburtstags der Deutschen Statistischen Gesellschaft (DstatG) möchte diese Ausgabe des Wirtschafts- und Sozialstatistischen Archivs dazu beitragen, Entwicklungstrends der Statistik aufzuzeigen. Was die Geschichte der DstatG angeht, so sei hier auf den Sammelband von Grohmann et al. (2011) verwiesen.

Ein Blick in die Zukunft, der die vergangenen Jahrzehnte extrapoliert, ist immer ein persönlicher Blick. In diesem Fall orientiert sich Rendtel (2011) an der Entwicklung der Rechner- und Speicherkapazitäten bei der Datenauswertung bzw. Datenhaltung, die auch neue Auswertungsmethoden eröffnet. Eng hiermit verknüpft ist die Entwicklung der Statistiksoftware. Hier wird nicht das Aussterben der Dinosaurier vom Typ SPSS vorhergesagt, wohl aber eine Symbiose von einem üppig wuchernden akademischen R-Dschungel und der geordneten Welt eines Standardpakets. Eine weitere Dimension der Betrachtung ist der Datenzugang bzw. der Typ der erhobenen Daten. Hier sind seit Gründung des Rats für Sozial- und Wirtschaftsdaten (RatSWD) und der Einrichtung von Forschungsdatenzentren dramatische Fortschritte erreicht worden. Im Rahmen des RatSWD wurde eine lebhafte Debatte über die Entwicklung des Datenzugangs und neue Datentypen geführt, die hier nur angerissen wird. Eine ausführliche Darstellung findet man in German Data Forum (2011).

In der öffentlichen Wahrnehmung wird „die Statistik“ häufig mit der Tätigkeit der Amtlichen Statistik identifiziert. Deshalb widmet Rendtel (2011) dem Verhältnis zur Amtlichen Statistik breiten Raum ein. Dass das Verhältnis von Amtlicher und akademischer Statistik nicht ungetrübt ist, ist vielerorts beschrieben worden, vgl. z.B. auch Rendtel (2008). Diese Diskussion wird hier unter dem Aspekt der Unabhängigkeit der Amtlichen Statistik und ihrer Organisation aufgenommen. Hier findet man einige

U. Rendtel ( $\square)$

Freie Universität Berlin, Berlin, Deutschland

e-mail: Ulrich.Rendtel@fu-berlin.de 
Vorschläge für eine Umgestaltung der Amtlichen Statistik, die für die beabsichtigte Novellierung des Bundesstatistikgesetzes interessant sein könnten.

Ein Blick in die Zukunft aus der Sicht des Statistischen Bundesamts wird in dem Beitrag von Egeler et al. (2011) gegeben. Der Blick geht von einer „öffentlichen Institution der Exekutive“ zur akademischen Wissenschaft mit großen Freiheiten in ihrer Arbeit jedoch ohne das ,schützende Korsett aus Behördenstruktur und Auskunftspflicht". Trotz dieser unterschiedlichen Rahmenbedingungen schildert der Beitrag von Egeler et al. die durchaus existierenden Begegnungspunkte von Amtlicher Statistik und Wissenschaft. Hier spielen die Forschungsdatenzentren von Bund und Ländern eine wichtige Scharnierfunktion. Interessant und neu sind die Ausführungen zur institutionellen Anbindung der Amtlichen Statistik in Deutschland. Diese ist durch die Anbindung an die Innenministerien des Bundes und der Länder gekennzeichnet. Wie ist diese Anbindung im Zusammenhang mit den vom Europäischen Statistischen System (ESS) geforderten Verhaltenskodex einer unabhängigen nationalen Statistik zu sehen? Das mahnende Beispiel ist hierbei Griechenland, das nur mit falschen Zahlen über seine Verschuldung den gewünschten Beitritt zur Eurozone schaffte. Die Frage der „unbedingten professionellen Unabhängigkeit“ der Amtlichen Statistik sei durchaus offen.

Die Entwicklung der europäischen Statistik zu einem eigenen Europäischen Statistik System skizziert Walter Radermacher (2011) in seinem Beitrag. Die Existenz europäischer Rechtsnormen im Datenschutz und ihr Verhältnis zu den nationalen Regelungen, das sind neue Aufgaben und Spannungsfelder, die einer Lösung bedürfen. Auch bei Radermacher ist der Code-of-Practice mit seinem Unabhängigkeitsgebot für die nationalen Statistikinstitute, wie die Statistikämter in der EU bezeichnet werden, ein zentrales Postulat.

Der letzte Beitrag in diesem Zukunftsheft ist zunächst der Rückschau gewidmet. Walter Krämer (2011) stellt die provozierende Frage, ob der Gebrauch statistischer Methodik gar zu einem Hemmschuh der Entwicklung in den Wirtschafts- und Sozialwissenschaften werden kann; also just zu dem Gegenteil von dem, was wir gerne über die Rolle der statistischen Methodik behaupten. Zitatenreich belegt Krämer, dass derartige Vorbehalte auch in der Medizin und Arzneimittelforschung aufgetaucht sind. Krämer hat viel Erfahrung mit einem irreführenden Umgang mit Statistik, vgl. z.B. Krämer (1991) „So lügt man mit Statistik“. In Krämers Beitrag wird der StatistikMissbrauch für Fortgeschrittene präsentiert: Die Hypothesenbildung nach Durchführung eines statistischen Tests. Dieses Vorgehen sei schlicht ein Hemmnis für die Substanzwissenschaften, so Krämer.

Die Beiträge dieses Zukunftheftes sind durchaus geeignet, Widerspruch zu provozieren. Ich lade Kritiker ein, ihre Einwendungen in einer der nächsten Ausgaben dieser Zeitschrift zu publizieren.

\section{Literatur}

Egeler R, Wöll Th, Zwick M (2011) Perspektiven für die amtliche Statistik. ASTA Wirt Sozialstat Archiv 5:4. doi:10.10.1007/s11943-012-0114-X

German Data Forum (RatSWD) (Hrsg) (2011) Building on progress-expanding the research infrastructure for social, economic and behavioral sciences, Budrich Univ. Press, Opladen 
Grohmann H, Krämer W, Steger A (Hrsg) (2011) Statistik in Deutschland - 100 Jahre Deutsche Statistische Gesellschaft. Springer, Heidelberg

Krämer W (1991) So lügt man mit Statistik. Campus Verlag, Frankfurt

Krämer W (2011) Das Signifikanztest-Ritual und andere Sackgassen des Fortschritts in der Statistik. ASTA Wirts Sozialstat Archiv 5(4). doi:10.1007/s11943-012-0110-1

Radermacher W (2011) Zahlen zählen Gedanken zur Zukunft der Amtlichen Statistik in Europa. ASTA Wirts Sozialstat Archiv. 5(4) doi:10.1007/s11943-012-0113-y

Rendtel U (2008) Statistikausbildung und Amtliche Statistik: Kritik und Perspektiven. ASTA Wirts Sozialstat Archiv 2:5-20

Rendtel U (2011) Die Zukunft der Statistik - eine persönliche Betrachtung. ASTA Wirts Sozialstat Archiv 5(4). doi:10.1007/s11943-012-0112-z 